\title{
Unified Model for Inflation and Dark Matter
}

\author{
Gabriel Zsembinszki \\ Grup de Física Teòrica and Institut de Física d'Altes Energies \\ Universitat Autònoma de Barcelona \\ 08193 Bellaterra, Barcelona, Spain
}

(Dated: February 5, 2008)

\begin{abstract}
We present a model which contains a new complex scalar field $\Psi$, interacting with a new real scalar field, $\chi$, and an effective potential whose symmetry is almost exact, being explicitly broken by Planck-scale effects. We study the possibility of relating inflation with the dark matter of the universe. We find that for exponentially small explicit breaking, the model accounts for a period of inflation in the early universe and gives a dark matter candidate particle.

PACS numbers: 98.80.-k, 98.80.Cq, 95.35.+d
\end{abstract}




\section{INTRODUCTION}

Cosmology has made in the last few years enormous progress, especially in the accuracy of observational data, which is taken using technologies more and more precise. While in the past not too remote it seemed almost inconceivable, nowadays we may even talk about "precise cosmology". This is why, in analogy with the Standard Model (SM) of particle physics, many physicists are already talking about a "standard model" of cosmology. As suggested by recent observations of type Ia supernovas (SNIa) [1], the matter power spectrum of large scale structure (LSS) [2] and anisotropy of the cosmic microwave background radiation $(\mathrm{CMB})[3]$, the universe is presently dominated by too types of mysterious fluids: dark energy (DE), which has negative pressure whose consequence is to accelerate the expansion of the universe, and dark matter (DM), which is non-relativistic non-baryonic matter, very weakly coupled to normal matter and that only has gravitational effects on it.

The most simple explanation for DE is a cosmological constant $\Lambda$, but it raises another problem because its expected value is many orders of magnitude larger than the value suggested by observations. Another possible explanation is the existence of a slowly rolling scalar field, called quintessence, which is displaced from the minimum of its potential and started to dominate the energy density of the universe recently.

The same observations indicate that the universe is isotropic and homogeneous at large scales and spatially flat, for which in the old cosmological picture there is no reasonable explanation. The most successful and simple solution to the flatness and homogeneity problems is given by inflation [4], which in its simplest version is defined as a short period of accelerated expansion of the early universe caused by a single dominating scalar field, the inflaton. In addition, inflation gives the most popular mechanism of generation of cosmological fluctuations, which were the seed for the structure formation in our universe.

Although the SM based on the gauge group $S U(3) \times S U(2) \times U(1)$ is a solid theoretical construction able to accommodate all existing empirical data, it leaves many deep questions unanswered when trying to explain the origin and nature of the new ingredients introduced by modern cosmology, such as, for example, the inflaton, the DE and the DM. Thus, there are reasons to believe that the SM is not the ultimate theory and one has to look for extensions of it. If we are able to discover a theory that indeed goes beyond the SM, it will probably contain new symmetries, either local, or global. 
A lot of effort has been done in studying global symmetries at high energies [5], especially in trying to clarify the issue of quantum coherence loss in the presence of wormholes. It was argued that the loss of coherence opens the possibility that currents associated with global symmetries are not exactly conserved. Even if incoherence is not observed in the presence of wormholes, it was argued that other interesting consequences may emerge, such as the appearance of operators that violate global symmetries, of arbitrary dimensions, induced by baby universe interactions. There are other reasons to expect that quantum gravity effects break global symmetries: global charges can be absorbed by black holes which may evaporate, "virtual black holes" may form and evaporate in the presence of a global charge, etc.

In this context, the authors of [6] argue that if global symmetries are broken by virtual black holes or topology changing effects, they have to be exponentially suppressed. In particular, in order to save the axion theory, the suppression factor should have an extremely small value $g<10^{-82}$. This suppression can be obtained in string theory, if the stringy mass scale is somewhat lower than the Planck-scale, $M_{\text {str }} \sim 2 \times 10^{18} \mathrm{GeV}$. Thus we expect to have an exponential suppression of the explicit breaking of global symmetries.

Even with such an extremely small explicit breaking, one can see that very interesting consequences may appear. In [7] (from now on Paper 1), it was shown that, when a global symmetry is spontaneously broken in the presence of a small explicit breaking, the resulting pseudo-Golstone boson (PGB) can be a DM particle. In [8] (from now on Paper 2), a similar study was made, but the purpose was to show that the resulting PGB could be a quintessence field explaining the present acceleration of the universe. In addition, based on the idea forwarded by Frieman and Rosenfeld [9], the model of Paper 2 also incorporated inflation. In this way, the two periods of accelerated expansion may have a common origin.

There is previous work related to explicit breaking of global symmetries [10] and to Planck-scale breaking [11]. Cosmological consequences of some classes of PGBs are discussed in $[12]$.

Here, we extend the model in Paper 1 to also include inflation. The way we do it is similar to the work in Paper 2, the difference being that here we want the resulting PGB to be a DM particle, in contrast with Paper 2 where it was a quintessence field. Our result is that the parameter of the explicit breaking should be exponentially suppressed, $g<10^{-30}$, as in Paper 1, but the level of suppressions is not that high as in the case of Paper 2, where 
a much smaller $g \sim 10^{-119}$ was needed in order for the PGB to explain DE. Inflation may occur here at scales as low as $V^{1 / 4} \sim 10^{10} \mathrm{GeV}$.

The paper is structured as follows: in section [II we make a short presentation of the model and then focus on the main features of it: inflation and dark matter. In section III we present our numerical results and, finally, in section [V we make a discussion and give the conclusions. Technical details are given in the Appendix A.

\section{THE MODEL}

The model is, basically, the same as in Paper 2, so we just recall it here shortly. It contains a new complex scalar field, $\Psi$, charged under a certain global $U(1)$ symmetry, interacting with a massive real scalar field, $\chi$, neutral under $U(1)$. It also contains a $U(1)$-symmetric potential

$$
V_{\mathrm{sym}}(\Psi, \chi)=\frac{1}{4} \lambda\left(|\Psi|^{2}-v^{2}\right)^{2}+\frac{1}{2} m_{\chi}^{2} \chi^{2}+\left(\Lambda^{2}-\frac{\kappa^{2}|\Psi|^{2} \chi^{2}}{4 \Lambda^{2}}\right)^{2}
$$

where $\lambda$ and $\kappa$ are coupling constants, $m_{\chi}$ and $\Lambda$ are some energy scales and $v$ is the $U(1)$ spontaneous symmetry breaking (SSB) scale.

The interaction term in (11) is of inverted hybrid type [13, 14] and can be realized in supersymmetry using a globally supersymmetric scalar potential [14]. However, in the present paper we are not preoccupied about the underlying theory in which this model can be realized, instead we only study the phenomenology of the potential (11).

Next, we allow terms in the potential that explicitly break $U(1)$. These terms are supposed to come from physics at the Planck-scale, and without knowledge of the exact theory at that scale, we introduce the most simple effective $U(1)$-breaking term [15]

$$
V_{\text {non-sym }}(\Psi)=-g \frac{1}{M_{\mathrm{P}}^{n-3}}|\Psi|^{n}\left(\Psi \mathrm{e}^{-\mathrm{i} \delta}+\Psi^{\star} \mathrm{e}^{\mathrm{i} \delta}\right)
$$

where $g$ is an effective coupling, $M_{\mathrm{P}} \equiv G_{\mathrm{N}}^{-1 / 2}$ is the Planck-mass and $n$ is an integer $(n>3)$.

Summarizing, our effective potential is

$$
V(\Psi, \chi)=V_{\text {sym }}(\Psi, \chi)+V_{\text {non-sym }}(\Psi)-C
$$

where $C$ is a constant that sets the minimum of the effective potential to zero.

By writing the field $\Psi$ as

$$
\Psi=\phi \mathrm{e}^{\mathrm{i} \tilde{\theta}}
$$


we envisage a model in which the radial field $\phi$ is the inflaton, while the angular field $\tilde{\theta}$ is associated with a DM particle.

Thus, in this paper we consider the possibility of having a unified model of inflation and DM, improving in this way the model presented in Paper 1. We also want to present a more detailed numerical analysis of the part regarding inflation, which could also apply to the inflationary model of Paper 2.

\section{A. Inflation}

We first revisit the conditions that should be accomplished by our model in order to correctly describe the inflationary period of expansion of the universe. Inflation is supposed to have occurred in the early universe, when the energies it contained were huge. Thus, the appropriate term to deal with when describing inflation is the symmetric term $V_{\text {sym }}$, while the non-symmetric one can be safely neglected, being many orders of magnitude smaller than $V_{\text {sym. }}$ Taking into account (41), we may write

$$
V_{\mathrm{sym}}(\phi, \chi)=\Lambda^{4}+\frac{1}{2} M_{\chi}^{2}(\phi) \chi^{2}+\frac{\kappa^{4} \phi^{4} \chi^{4}}{16 \Lambda^{4}}+\frac{1}{4} \lambda\left(\phi^{2}-v^{2}\right)^{2}-C
$$

where $M_{\chi}^{2}(\phi) \equiv m_{\chi}^{2}-\kappa^{2} \phi^{2}$, and we have also included the constant $C$. As commented above, $\phi$ is the inflaton field and $\chi$ is an auxiliary field that assists $\phi$ to inflate.

We assume that initially the fields $\phi$ and $\chi$ are in the vicinity of the origin of the potential, $\phi=\chi=0$. At that point, the first derivatives of the potential are zero in both $\phi-$ and $\chi$-directions, but the second derivatives have opposite signs:

$$
\begin{aligned}
& \left.\frac{\partial^{2} V_{\mathrm{sym}}(\phi, \chi)}{\partial \phi^{2}}\right|_{\phi, \chi=0}=-\lambda v^{2}<0 \\
& \left.\frac{\partial^{2} V_{\mathrm{sym}}(\phi, \chi)}{\partial \chi^{2}}\right|_{\phi, \chi=0}=m_{\chi}^{2}>0 .
\end{aligned}
$$

This means that $\chi$ remains trapped at the false minimum in $\chi$-direction of the potential, $\chi=0$, while $\phi$ becomes unstable and can roll down in the direction given by $\chi=0$. If the potential in $\phi$-direction is sufficiently flat, $\phi$ can have a slow-roll and produce inflation. This regime lasts until the curvature in $\chi$-direction changes sign and inflation has a sudden end through the instability of $\chi$, which triggers a waterfall regime and both fields rapidly evolve towards the absolute minimum of the potential. The critical point where inflation 
ends is given by the condition

$$
M_{\chi}^{2}(\phi)=m_{\chi}^{2}-\kappa^{2} \phi^{2}=0
$$

so that during inflation $\phi<\phi_{\mathrm{c}}=\frac{m_{\chi}}{\kappa} \lesssim v$.

The constraints related to the inflationary aspects of the model are the same of Paper 2. Let us just summarize them here.

- vacuum energy of field $\chi$ should dominate: $\frac{1}{4} \lambda v^{4} \ll \Lambda^{4}$

- small $\phi$-mass as compared to $\chi$-mass: $\left|m_{\phi}^{2}\right|=\lambda v^{2} \ll m_{\chi}^{2} \lesssim \kappa^{2} v^{2}$

- slow-roll conditions: $\epsilon \equiv \frac{1}{16 \pi}\left(\frac{V^{\prime}}{V}\right)^{2} \ll 1,|\eta| \equiv\left|\frac{1}{8 \pi} \frac{V^{\prime \prime}}{V}\right| \ll 1$

- rapid variation of $M_{\chi}^{2}(\phi)$ at the critical point: $\left|\Delta M_{\chi}^{2}\left(\phi_{\mathrm{c}}\right)\right|>H^{2}$

- fast roll of $\phi$ after $\chi$ gets to its minimum: large $\left.\left(\partial V_{\mathrm{sym}} / \partial \phi\right)\right|_{\chi_{\min }}$

These conditions have to be satisfied in order for the hybrid inflationary mechanism to work. There are other constraints related to fairly precise observational data:

- sufficient number of e-folds of inflation $N(\phi)=(8 \pi) / M_{\mathrm{P}}^{2} \int_{\phi_{\text {end }}}^{\phi}\left(V_{\text {sym }} / V_{\text {sym }}^{\prime}\right) \mathrm{d} \phi$ in order to solve the flatness and the horizon problems. The required number depends on the inflationary scale and on the reheating temperature, and is usually comprised between 35 for low-scale inflation and 60 for GUT-scale inflation

- the amplitude of the primordial curvature power-spectrum produced by quantum fluctuations of the inflaton field should fit the observational data $[3], \mathcal{P}_{\mathcal{R}}{ }^{1 / 2} \simeq 4.86 \times 10^{-5}$

- the spectral index $n_{\mathrm{s}}$ should have the right value suggested by observations of the $\mathrm{CMB}[3], n_{\mathrm{s}}=0.951_{-0.019}^{+0.015}$ (provided tensor-to-scalar ratio $r \ll 1$ ).

Combining all the above constraints we obtain the following final relations that should be satisfied by the parameters of our model: $\lambda \ll \kappa^{2}$ and $v<M_{\mathrm{P}}$. We also obtain the dependence of some of the model parameters on the SSB scale $v$ (for more details, see Paper 2 and the Appendix (A). These will be used in section III for a numerical study. The range of values of the scale $v$ will be fixed by the requirement that $\theta$ is a DM candidate. 


\section{B. Dark matter}

As stated above, our idea is that $\theta$, the PGB that appears after the SSB of $U(1)$ in the presence of a small explicit breaking, can play the role of a DM particle. Thus, after the end of inflation, $\theta$ finds itself in a potential given by the term $V_{\text {non-sym }}$

$$
V_{\text {non-sym }}(\phi, \theta)=-2 g \frac{\phi^{n+1}}{M_{P}^{n-3}} \cos \tilde{\theta}
$$

where (4) has been used in (2) and the change of variables $\tilde{\theta} \longrightarrow \tilde{\theta}+\delta$ has been made. In Paper 1 it was shown that for exponentially small $g$ the evolutions of the two components of $\Psi$ are completely separated, so that we expect $\theta$-oscillations to start long after $\phi$ has settled down at its vacuum expectation value (vev),

$$
\langle\phi\rangle \simeq M_{P}^{1 / 3} v^{2 / 3}
$$

A detailed study of the cosmology of the $\theta$-particle was made in Paper 1 , for the lowest possible value $n=4$. We do not want to enter into details here, but just to make use of the results of that work to obtain the values of the parameters of our model. The only difference here is the fact that the vev of the radial field $\phi$ is different from $v$, so that the constraints

obtained in Paper 1 on $v$ will apply here on $\langle\phi\rangle$. This will also affect the angular field $\tilde{\theta}$, which is here normalized as $\tilde{\theta} \equiv \theta /\langle\phi\rangle$.

Due to the small explicit breaking of the $U(1)$ symmetry, $\theta$ acquires a mass which depends on both $g$ and $\langle\phi\rangle$

$$
m_{\theta}^{2}=2 g\left(\frac{\langle\phi\rangle}{M_{P}}\right)^{3} M_{P}^{2}
$$

and this is why we should find constraints on both $\langle\phi\rangle$ and $g$ in order for $\theta$ to be a suitable DM candidate. The constraints that should be imposed come from various astrophysical and cosmological considerations:

- $\theta$ should be a stable particle, with lifetime $\tau_{\theta}>t_{0}$, where $t_{0}$ is the universe's lifetime

- its present density should be comparable to the present DM density $\Omega_{\theta} \sim \Omega_{D M} \sim 0.25$

- it should not allow for too much energy loss and rapid cooling of stars [16]

- although stable, $\theta$ may be decaying at present, and its decay products should not distort the diffuse photon background 
In Paper 1, all these constraints have been studied in detail. Because $\theta$ is massive, it can decay into two photons or two fermions, depending on its mass. The lifetime of $\theta$ depends on the effective coupling to the two photons/fermions and on its mass, which in turn depends on the two parameters $\langle\phi\rangle$ and $g$. It was shown that for the interesting value of $\langle\phi\rangle$ and $g$ for which $\theta$ can be DM, the resulting $\theta$-mass has to be $m_{\theta}<20 \mathrm{eV}$, so the only decaying channel is into two photons.

There are different mechanism by which $\theta$ particles can be produced, as explained in Paper 1: (a) thermal production in the hot plasma, and (b) non-thermal production by $\theta$-field oscillations and by the decay of cosmic strings produced in the SSB. All these may contribute to the present energy density of $\theta$ particles, which was computed in Paper 1. By requiring it to be comparable to the present DM energy density of the universe, we obtain a curve in the space of parameters $\langle\phi\rangle$ and $g$, illustrated in Fig 1 as the line labelled "DM".

In Paper 1 it was argued that there are some similarities between our $\theta$ particle and the QCD axion [17]. This is why when investigating its production in stars, we can apply similar constraints. The strongest one comes from the fact that $\theta$ may be produced in stars and constitute a novel energy loss channel, and these considerations put a limit on $\langle\phi\rangle$, but not on $g$

$$
\langle\phi\rangle>3.3 \times 10^{9} \mathrm{GeV}
$$

Another aspect about $\theta$ is that, although stable, a small fraction of its population may be decaying today and the resulting photons may produce distortions of the diffuse photon background. Thus, the calculated photon flux coming from $\theta$-decay is constraint to be less than the observed flux (see Paper 1 for details).

By combining astrophysical and cosmological constraints, we can obtain the interesting values for $\langle\phi\rangle$ and $g$ for which $\theta$ is stable and its density is comparable to the DM density. These values can be easily read from Fig,1, being situated along the line labelled "DM". Note that there is an upper limit on $g$, which corresponds to a lower limit on $\langle\phi\rangle$, i.e.

$$
g<10^{-30},\langle\phi\rangle>10^{11} \mathrm{GeV} .
$$

These also put a lower limit on the value of $U(1)$ breaking scale,

$$
v \sim\left(\frac{\langle\phi\rangle^{3}}{M_{\mathrm{P}}}\right)^{1 / 2}>10^{7} \mathrm{GeV} .
$$




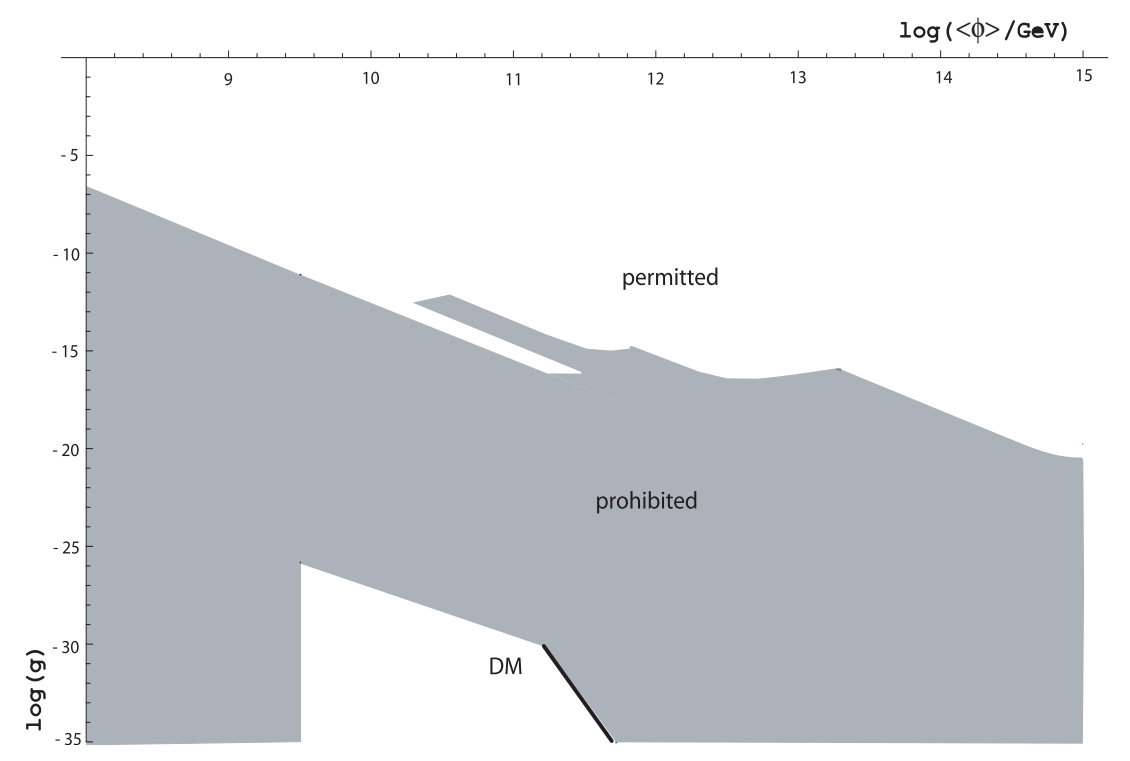

FIG. 1: Permitted and prohibited regions in the $(\langle\phi\rangle, g)$ - plane, taken from Paper 1. The interesting points are those which are near the line labelled "DM".

Finally, according to the study made in Paper 1 , for value $\langle\phi\rangle<7.2 \times 10^{12} \mathrm{GeV}, \theta$ particles can be produced both thermally and non-thermally, but in the region characterized by $\langle\phi\rangle>10^{11} \mathrm{GeV}$, the dominant energy density corresponds to non-thermally produced $\theta$ particles. Moreover, for $\langle\phi\rangle>7.2 \times 10^{12} \mathrm{GeV}$, only non-thermal production is possible.

\section{NUMERICAL RESULTS}

The constraints enumerated in subsection $\llbracket \mathrm{IA}$ will determine the values of some of the model parameters. Our scope is to make a general analysis of how these parameters depend on $v$. For $\lambda$ we obtain an exact formula (see Appendix $\mathrm{A}$ )

$$
\lambda=4.4 \times 10^{-12} \frac{\phi_{0}^{2}\left(v^{2}-\phi_{0}^{2}\right)^{2}}{\left(v^{2}-3 \phi_{0}^{2}\right)^{3}}
$$

where $\phi_{0}$ is the value of the inflaton field when the scale $k=0.002 \mathrm{Mpc}^{-1}$ crossed the horizon during inflation and its $v$-dependence can be obtained numerically, see the Appendix A,

The exact formulae for the other parameters are too complicate to be shown here. Nevertheless, things get simplified in the limit $\lambda v^{4} \ll \Lambda^{4}\left(\equiv v \ll M_{\mathrm{P}}\right)$, for which we obtain

$$
\Lambda \simeq 1.6 \times 10^{-3}\left[\frac{\phi_{0}^{2}\left(v^{2}-\phi_{0}^{2}\right)^{2}}{\left(v^{2}-3 \phi_{0}^{2}\right)^{2}}\right]^{1 / 4} M_{\mathrm{P}}^{1 / 2} \sim \lambda^{1 / 4} v^{1 / 2} M_{\mathrm{P}}^{1 / 2}
$$


and

$$
C \simeq \frac{3}{4} \Lambda^{4}\left(\lambda v^{4} / \Lambda^{4}\right)^{1 / 3} \ll \Lambda^{4}
$$

We notice that $C$ can be neglected, as compared to $\Lambda^{4}$, in this limit.

The coupling $\kappa$ is only constrained by the condition $\lambda \ll \kappa^{2}$, so that it can have any arbitrary value satisfying this inequality. In our numerical study we took the value $\kappa=10^{-2}$. The mass of $\chi$, namely $m_{\chi}$, can have any arbitrary value satisfying $m_{\chi}<\kappa v$, but for the sake of simplicity we set it to $m_{\chi}=\kappa v / 2$, without loss of generality.

In Fig 2 we display some graphics with the $v$-dependence of relevant parameters of our model. In Fig.2(a) we plot the numerical results for $\phi_{0}(v)$, which are then used to produce the other graphics. From Fig,2(b), one can see that $\lambda$ does not vary too much with $v$ and its values are around $10^{-13}$ for a large range of $v$. We also notice that the other parameters grow as different powers of $v$. For example in Fig.2(c), $\Lambda$, which sets the inflationary scale, varies as $v^{1 / 2}$ from $\sim 10^{10} \mathrm{GeV}$, for $v=10^{7} \mathrm{GeV}$, to $\sim 10^{16} \mathrm{GeV}$, for $v \sim M_{\mathrm{P}}$, i.e., from a relatively low-scale to a GUT-scale inflation. In Fig.2(d) are represented in the same graphic the values of $\Lambda^{4}, C$ and $\lambda v^{4}$ to confirm that, in the limit $v \ll M_{\mathrm{P}}$, one can use the approximation $\lambda v^{4} \ll C \ll \Lambda^{4}$, while for $v \lesssim M_{\mathrm{P}}$ the three terms become of the same order and the above approximation is not valid anymore. In Fig.2(e) and (f) we give additional results, such as the number of "observable" inflation $N$ and the tensor-to-scalar ratio $r \equiv 16 \epsilon$.

In particular, for the lowest possible value $v=10^{7} \mathrm{GeV}$, we get $N \simeq 47$ e-folds of inflation, and a very tiny value for the tensor-to-scalar ratio, $r \sim 10^{-27}$, making the detection of gravitational waves a practically impossible task. We specify that the spectral index $n_{\mathrm{s}} \simeq$ 0.95 and the amplitude of curvature perturbations, $\mathcal{P}_{\mathcal{R}}{ }^{1 / 2} \simeq 4.86 \times 10^{-5}$ for all $v$.

We also notice that for $v \sim M_{\mathrm{P}}$, we recover the inflationary scenario proposed in Paper 2, where $\theta$ was a quintessence field. The numerical analysis presented here can also be applied to that model, and one obtains $\langle\phi\rangle \sim v, N \sim 56$ e-folds of inflation and the more interesting result $r \sim 10^{-3}-10^{-4}$, which makes gravitational waves detection more plausible in the future.

\section{DISCUSSIONS AND CONCLUSIONS}

In this work, we have presented a model that is able to describe inflation and dark matter

in a unified scenario, by introducing a new complex scalar field $\Psi=\phi \exp (\mathrm{i} \tilde{\theta})$ interacting 


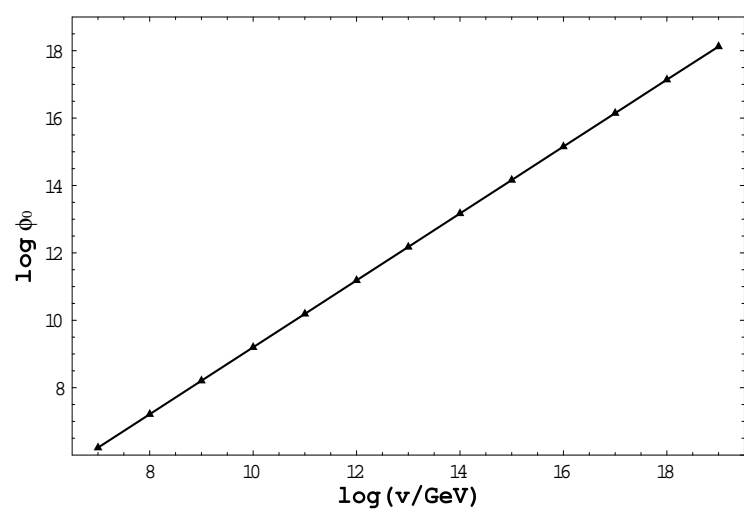

(a)

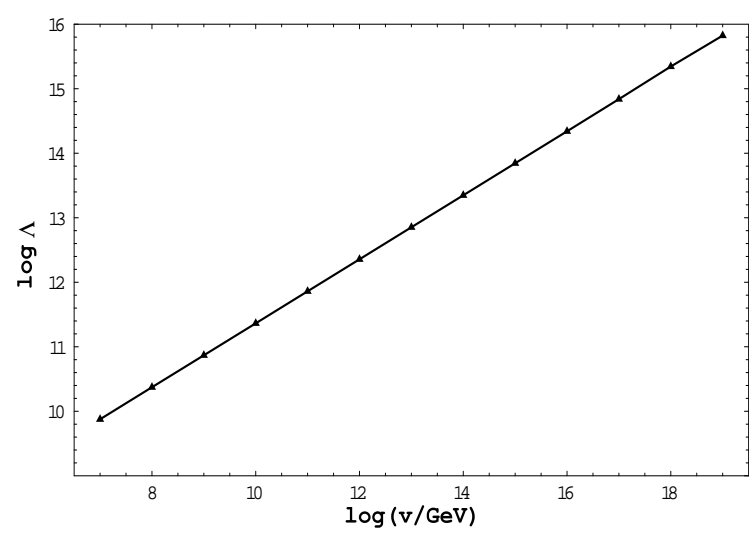

(c)

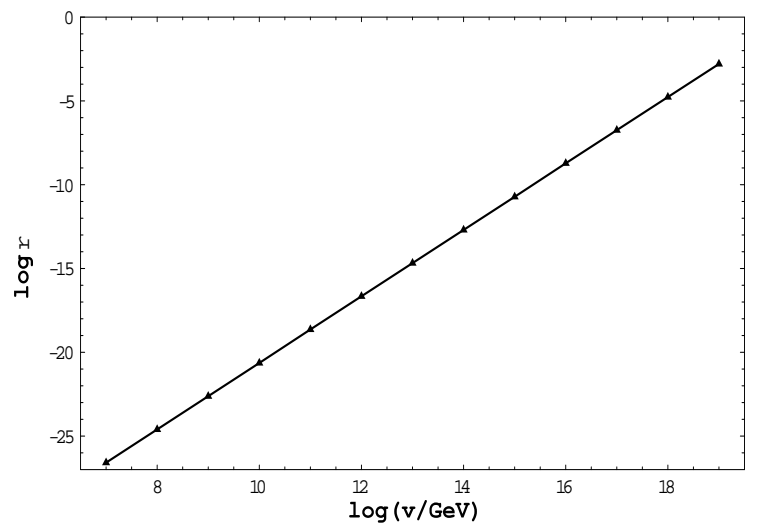

(e)

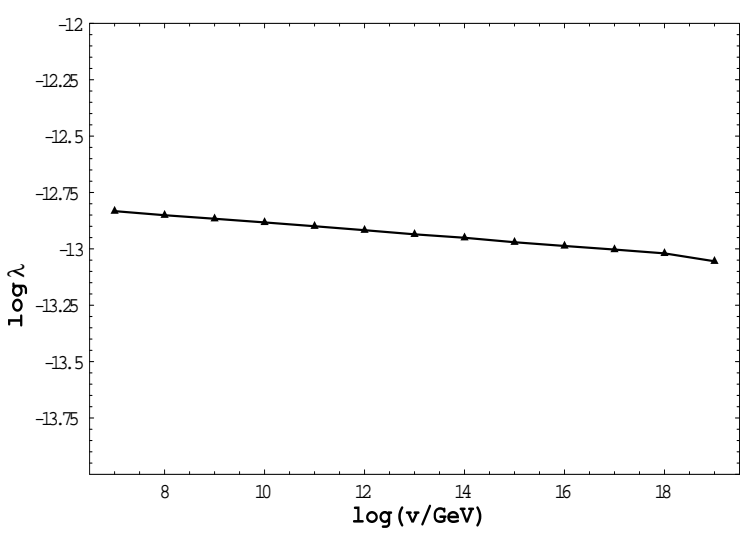

(b)

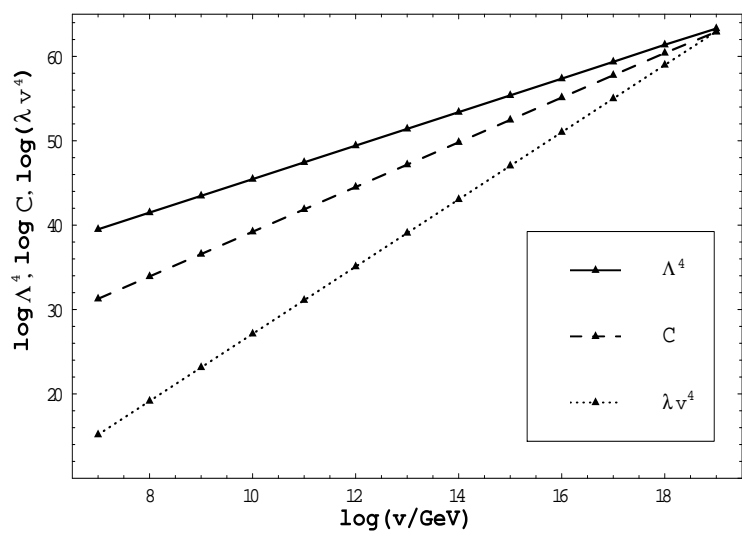

(d)

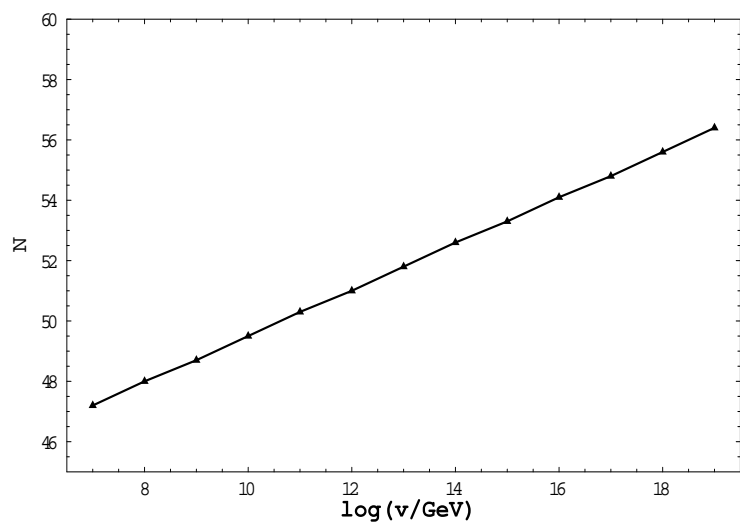

(f)

FIG. 2: $v$-dependence of various parameters: (a)-the inflaton field value corresponding to the moment when the scale $k_{0}=0.002 \mathrm{Mpc}^{-1}$ left the horizon, $\phi_{0}(v)$; (b)-the inflaton self-coupling constant, $\lambda(v)$; (c)-the scale of inflation, $\Lambda(v)$; (d)-comparison between $\Lambda^{4}(v), C(v)$ and $\lambda v^{4}$, which tend to be of the same order for $v \sim M_{\mathrm{P}}$; (e)-the tensor-to-scalar ratio, $r(v)$; (f)-the number of e-folds of inflation that occur between the largest observable scale left the horizon and the end of inflation, $N(v)$. 
with a real scalar, $\chi$, and a potential invariant under certain global $U(1)$ symmetry. We allowed for a small explicit breaking term in the effective potential that is due to Planck-scale physics and investigated the possibility that $\phi$ is the inflaton and $\theta$ a dark matter particle. The corresponding constraints have been enumerated in subsections II A and II B.

In this way, we improve the model of Paper 1 , where $\theta$ was a DM particle, but the model did not include inflation. The results of Paper 1 are used here in subsection IB, For the part regarding inflation in our model, in subsection $\amalg \mathrm{A}$ we make a similar analysis as in Paper 2, which also improves Paper 1 by incorporating inflation, but the difference is that there $\theta$ was a quintessence field. The numerical analysis we present in section [II extrapolates between the two scales considered in the models of Paper 1 and Paper 2.

In the present numerical study, we used the value $\kappa=10^{-2}$, and we chose $m_{\chi}=\kappa v / 2$ for simplicity. We observe that a tiny value is needed for $\lambda \sim 10^{-13}$, in order to generate the correct values of the amplitude of curvature perturbations and of the spectral index. We have no possible theoretical explanation for justifying this small $\lambda$-value, but this is a common problem of most of the inflationary models. Although we make a general numerical analysis to see how the parameters depend on the SSB scale $v$, we are finally interested in the value for which the angular field $\theta$ is a DM candidate, $v \ll M_{\mathrm{P}}$.

Notice that for $v \ll M_{\mathrm{P}}$, the vev of the inflaton field $\phi$ is different from $v$ and is approximately given by $\langle\phi\rangle \simeq v^{2 / 3} M_{\mathrm{P}}^{1 / 3} \neq v$, while for $v \sim M_{\mathrm{P}}$ they tend to be of the same order, $\langle\phi\rangle \sim O(v)$.

We included explicit $U(1)$-breaking terms in the potential and studied the possibility that the resulting PGB, $\theta$, could be a DM particle. We found in Eq.(13) that the effective $g$ coupling related to the explicit breaking should be exponentially suppressed, $g<10^{-30}$. This confirms our expectations commented in the Introduction, that the effect of Planckscale physics in breaking global symmetries should be exponentially suppressed [6]. With the extreme values $g=10^{-30}$ and $v=10^{7} \mathrm{GeV}$, the mass of $\theta$ is fully determined, $m_{\theta} \sim 15$ $\mathrm{eV}$.

It would be interesting to investigate reheating in our model to determine the exact reheating temperature $T_{\mathrm{rh}}$, and also to provide a specific mechanism for producing SM particles, but this goes beyond the scope of our paper.

As a final comment, we would like to add that such a strong suppression of $g$ may be avoided if, for some reason, $n=7$ and all smaller values prohibited. In this case, one obtains 
$g$ of $O(1)$, but then one should find an argument why $n$ cannot be smaller than 7 .

\section{APPENDIX A}

Here we give the details of how to obtain the parameters related to inflation in our model. We base our analysis on 3 constraints, which are:

1. The number of e-folds of inflation between a given scale $k$ crosses the horizon and the end of inflation is given by [18]

$$
N \simeq 62-\ln \frac{k}{a_{0} H_{0}}-\ln \left(\frac{10^{16} \mathrm{GeV}}{V_{\mathrm{sym}}\left(\phi_{0}, 0\right)^{1 / 4}}\right)+\frac{1}{3} \ln \left(\frac{T_{\mathrm{rh}}}{V_{\mathrm{sym}}\left(\phi_{0}, 0\right)^{1 / 4}}\right)
$$

where $T_{\mathrm{rh}}$ is the reheating temperature and $a_{0} / k=H_{0}^{-1} \simeq 4000 \mathrm{Mpc}$ is the biggest observable scale. A scale of interest is $k_{0}=0.002 \mathrm{Mpc}^{-1}$, for which we have reliable observational data [3]. The number of e-folds corresponding to $k_{0}$ can be expressed in terms of the inflaton field $\phi_{0}$

$$
\begin{aligned}
N\left(\phi_{0}\right) & =\frac{8 \pi}{M_{\mathrm{P}}^{2}} \int_{\phi_{\text {end }}}^{\phi_{0}} \frac{V_{\text {sym }}}{V_{\text {sym }}^{\prime}} \mathrm{d} \phi \\
& =\frac{\pi}{M_{\mathrm{P}}^{2}}\left[\frac{4\left(\Lambda^{4}-C\right)}{\lambda v^{2}} \ln \frac{\left(v^{2}-\phi_{0}^{2}\right) \phi_{\text {end }}^{2}}{\left(v^{2}-\phi_{\text {end }}^{2}\right) \phi_{0}^{2}}+v^{2} \ln \frac{\phi_{\text {end }}^{2}}{\phi_{0}^{2}}-\left(\phi_{\text {end }}^{2}-\phi_{0}^{2}\right)\right] .
\end{aligned}
$$

From now on we set $T_{\mathrm{rh}}=10^{9} \mathrm{GeV}$ and $\phi_{\text {end }}=v / 2\left(\equiv m_{\chi}=\kappa v / 2\right)$, for simplicity. By equating the two expressions (A1) and (A2) for $N\left(\phi_{0}\right)$, we finally obtain

$$
\begin{aligned}
60 & -\ln \frac{10^{16} \mathrm{GeV}}{\left[\Lambda^{4}-C+\frac{1}{4} \lambda\left(v^{2}-\phi_{0}^{2}\right)^{2}\right]^{1 / 4}}+\frac{1}{3} \ln \frac{10^{9} \mathrm{GeV}}{\left[\Lambda^{4}-C+\frac{1}{4} \lambda\left(v^{2}-\phi_{0}^{2}\right)^{2}\right]^{1 / 4}} \\
& =\frac{\pi}{M_{\mathrm{P}}^{2}}\left[\frac{4\left(\Lambda^{4}-C\right)}{\lambda v^{2}} \ln \frac{v^{2}-\phi_{0}^{2}}{3 \phi_{0}^{2}}+v^{2} \ln \frac{v^{2}}{4 \phi_{0}^{2}}-\left(\frac{v^{2}}{4}-\phi_{0}^{2}\right)\right] .
\end{aligned}
$$

2. The amplitude of the curvature perturbations $\mathcal{P}_{\mathcal{R}}{ }^{1 / 2}$ has the observed value $\mathcal{P}_{\mathcal{R}}{ }^{1 / 2} \simeq$ $4.86 \times 10^{-5}$ corresponding to the scale $k_{0}[3]$. This means that

$$
\begin{aligned}
\mathcal{P}_{\mathcal{R}}{ }^{1 / 2} & =\sqrt{\frac{128 \pi}{3}}\left|\frac{V_{\mathrm{sym}}\left(\phi_{0}, 0\right)^{3 / 2}}{M_{\mathrm{P}}^{3} V_{\mathrm{sym}}^{\prime}\left(\phi_{0}, 0\right)}\right| \\
& =\sqrt{\frac{128 \pi}{3}} \frac{\left[\Lambda^{4}-C+\frac{1}{4} \lambda\left(v^{2}-\phi_{0}^{2}\right)^{2}\right]^{3 / 2}}{\lambda M_{\mathrm{P}}^{3} \phi_{0}\left(v^{2}-\phi_{0}^{2}\right)} \simeq 4.86 \times 10^{-5} .
\end{aligned}
$$


3. The value of the spectral index $n_{\mathrm{s}} \simeq 1+2 \eta$ should have a value close to $n_{\mathrm{s}}=0.95$ for the same scale $k_{0}=0.002 \mathrm{Mpc}^{-1}$, where $\eta=\left(M_{\mathrm{P}}^{2} / 8 \pi\right)\left(V_{\mathrm{sym}}^{\prime \prime} / V_{\mathrm{sym}}\right)$ is a slow-roll parameter. This becomes

$$
2 \eta=\frac{-\lambda M_{\mathrm{P}}^{2}\left(v^{2}-3 \phi_{0}^{2}\right)}{4 \pi\left[\Lambda^{4}-C+\frac{1}{4} \lambda\left(v^{2}-\phi_{0}^{2}\right)^{2}\right]} \simeq-0.05 .
$$

One can see that by combining equations (A4) and (A5) one obtains an expression for $\lambda$ in terms of $\phi_{0}$ and $v$

$$
\lambda=4.4 \times 10^{-12} \frac{\phi_{0}^{2}\left(v^{2}-\phi_{0}^{2}\right)^{2}}{\left(v^{2}-3 \phi_{0}^{2}\right)^{3}} .
$$

Next, by replacing (A6) into (A5) one obtains

$$
\Lambda^{4}-C=4.4 \times 10^{-12} \frac{\phi_{0}^{2}\left(v^{2}-\phi_{0}^{2}\right)^{2}}{\left(v^{2}-3 \phi_{0}^{2}\right)^{2}}\left[\frac{5}{\pi} M_{\mathrm{P}}^{2}-\frac{\left(v^{2}-\phi_{0}^{2}\right)^{2}}{4\left(v^{2}-3 \phi_{0}^{2}\right)}\right] .
$$

Finally, by introducing (A6) and (A7) into (A3), one obtains an equation which relates $\phi_{0}$ and $v$. We solved it numerically for a few $v$-values in the interval $\left(10^{7}-10^{19}\right) \mathrm{GeV}$ and we obtained the corresponding values for $\phi_{0}$, which are shown in Fig[1(a). Once we have $\phi_{0}(v)$, we can turn back to (A6) and (A7) and find the values of $\lambda$ and $\Lambda^{4}-C$, respectively.

Still, we would like to find $\Lambda$ and $C$, separately. This can be done by requiring that the absolute minimum of $V_{\mathrm{sym}}(\phi, \chi)$ is equal to zero. The position of the absolute minimum is given by the following conditions

$$
\begin{aligned}
& \frac{\partial V_{\mathrm{sym}}(\phi, \chi)}{\partial \phi}=-\lambda v^{2} \phi+\lambda \phi^{3}-\kappa^{2} \phi \chi^{2}+\frac{\kappa^{4} \phi^{3} \chi^{4}}{4 \Lambda^{4}}=0, \frac{\partial^{2} V_{\mathrm{sym}}(\phi, \chi)}{\partial \phi^{2}}>0 \\
& \frac{\partial V_{\mathrm{sym}}(\phi, \chi)}{\partial \chi}=m_{\chi}^{2} \chi-\kappa^{2} \phi^{2} \chi+\frac{\kappa^{4} \phi^{4} \chi^{3}}{4 \Lambda^{4}}=0, \frac{\partial^{2} V_{\mathrm{sym}}(\phi, \chi)}{\partial \chi^{2}}>0 .
\end{aligned}
$$

Solving the above equation system, one can obtain $\phi_{\min }$ and $\chi_{\min }$. We do not show here the analytical solutions because they are very complicated. From the condition $V_{\mathrm{sym}}\left(\phi_{\mathrm{m}}, \chi_{\mathrm{m}}\right)=$ 0 , one can obtain a relation between $C$ and $\Lambda$. With this, going back to equation (A7), one obtains the dependence $\Lambda(v)$ and subsequently $C(v)$. The results we obtained are shown in Fig! (c) and (d), respectively.

Things become much simpler in the limit $v \ll M_{\mathrm{P}}$. As shown in Fig:1(d), when $v \ll M_{\mathrm{P}}$, the following relations are satisfied

$$
\lambda v^{4} \ll C \ll \Lambda^{4} .
$$


In this case, from (A5) we obtain

$$
\Lambda^{4} \simeq \frac{5}{\pi} \lambda M_{\mathrm{P}}^{2}\left(v^{2}-3 \phi_{0}^{2}\right) \sim \lambda v^{2} M_{\mathrm{P}}^{2}
$$

and the solutions of (A8) and (A9) become very simple

$$
\phi_{\mathrm{m}} \simeq \frac{v^{1 / 3} \Lambda^{2 / 3}}{\lambda^{1 / 6}} \sim v^{2 / 3} M_{\mathrm{P}}^{1 / 3}, \quad \chi_{\mathrm{m}} \simeq \frac{2 \lambda^{1 / 6} \Lambda^{4 / 3}}{\kappa v^{1 / 3}} .
$$

With the above expressions, the approximate solution for $C$ is also very simple

$$
C \simeq \frac{3}{4} \Lambda^{4}\left(\frac{\lambda v^{4}}{\Lambda^{4}}\right)^{1 / 3} \sim \Lambda^{4}\left(\frac{v}{M_{\mathrm{P}}}\right)^{2 / 3} \sim \lambda v^{4}\left(\frac{M_{\mathrm{P}}}{v}\right)^{4 / 3}
$$

where we made use of (A11). This also helps us understand why the following inequalities $\lambda v^{4} \ll C \ll \Lambda^{4}$ are satisfied for $v \ll M_{\mathrm{P}}$.

In the same limit $\left(v \ll M_{\mathrm{P}}\right)$, we get simple expressions for the $v$-dependence of the number of e-folds of observable inflation, $N(v) \propto \ln v$, and of the tensor-to-scalar ratio, $r(v) \propto v^{2} / M_{\mathrm{P}}^{2}$.

\section{ACKNOWLEDGMENTS}

I thank Eduard Massó for useful discussions and comments. This work is supported by the Spanish grant FPA-2005-05904 and by Catalan DURSI grants 2005SGR00916 and 2003 FI00138.

[1] A. G. Riess et al. [Supernova Search Team Collaboration], Astrophys. J. 607, 665 (2004) arXiv:astro-ph/0402512; R. A. Knop et al. [Supernova Cosmology Project Collaboration], Astrophys. J. 598, 102 (2003) arXiv:astro-ph/0309368.

[2] M. Tegmark et al. [SDSS Collaboration], Phys. Rev. D 69, 103501 (2004) arXiv:astro-ph/0310723.

[3] D. N. Spergel et al., arXiv:astro-ph/0603449.

[4] A. H. Guth, Phys. Rev. D 23, 347 (1981).

[5] T. Banks, Physicalia 12, 19 (1990); S. B. Giddings and A. Strominger, Nucl. Phys. B 307, 854 (1988); S. R. Coleman, Nucl. Phys. B 310, 643 (1988); G. Gilbert, Nucl. Phys. B 328, 159 (1989). 
[6] R. Kallosh, A. D. Linde, D. A. Linde and L. Susskind, Phys. Rev. D 52, 912 (1995) arXiv:hep-th/9502069].

[7] E. Masso, F. Rota and G. Zsembinszki, Phys. Rev. D 70, 115009 (2004) [arXiv:hep-ph/0404289.

[8] E. Masso and G. Zsembinszki, JCAP 0602, 012 (2006) arXiv:astro-ph/0602166.

[9] R. Rosenfeld and J. A. Frieman, JCAP 0509, 003 (2005) arXiv:astro-ph/0504191.

[10] C. T. Hill and G. G. Ross, Phys. Lett. B 203, 125 (1988); C. T. Hill and G. G. Ross, Nucl. Phys. B 311, 253 (1988).

[11] M. Lusignoli, A. Masiero and M. Roncadelli, Phys. Lett. B 252, 247 (1990); S. Ghigna, M. Lusignoli and M. Roncadelli, Phys. Lett. B 283, 278 (1992); D. Grasso, M. Lusignoli and M. Roncadelli, Phys. Lett. B 288, 140 (1992).

[12] C. T. Hill, D. N. Schramm and J. N. Fry, Comments Nucl. Part. Phys. 19, 25 (1989); A. K. Gupta, C. T. Hill, R. Holman and E. W. Kolb, Phys. Rev. D 45, 441 (1992); J. A. Frieman, C. T. Hill and R. Watkins, Phys. Rev. D 46, 1226 (1992); J. A. Frieman, C. T. Hill, A. Stebbins and I. Waga, Phys. Rev. Lett. 75, 2077 (1995) arXiv:astro-ph/9505060.

[13] B. A. Ovrut and P. J. Steinhardt, Phys. Rev. Lett. 53, 732 (1984).

[14] D. H. Lyth and E. D. Stewart, Phys. Rev. D 54, 7186 (1996) arXiv:hep-ph/9606412.

[15] R. Holman, S. D. H. Hsu, T. W. Kephart, E. W. Kolb, R. Watkins and L. M. Widrow, Phys. Lett. B 282, 132 (1992) arXiv:hep-ph/9203206; M. Kamionkowski and J. March-Russell, Phys. Lett. B 282, 137 (1992) arXiv:hep-th/9202003; S. M. Barr and D. Seckel, Phys. Rev. D 46, 539 (1992).

[16] For a comprehensive review, see G. G. Raffelt, Stars As Laboratories For Fundamental Physics (University of Chicago Press, Chicago, 1996).

[17] S. Weinberg, Phys. Rev. Lett. 40, 223 (1978); F. Wilczek, Phys. Rev. Lett. 40, 279 (1978).

[18] A. R. Liddle and D. H. Lyth, Phys. Rept. 231, 1 (1993) arXiv:astro-ph/9303019]; J. E. Lidsey, A. R. Liddle, E. W. Kolb, E. J. Copeland, T. Barreiro and M. Abney, Rev. Mod. Phys. 69, 373 (1997) arXiv:astro-ph/9508078]; D. H. Lyth and A. Riotto, Phys. Rept. 314, 1 (1999) arXiv:hep-ph/9807278; A. R. Liddle and S. M. Leach, Phys. Rev. D 68, 103503 (2003) arXiv:astro-ph/0305263. 